\title{
Use of chip-based PCR for 3D absolute digital quantification of microRNAs molecules for the non- invasive diagnostic screening of human colon cancer in stool
}

\author{
Farid E Ahmed* \\ DGEM Tox Labs, Institute for Research in Biotechnology, USA
}

There is no validated approach to screen for colon cancer (CC) quantitativley on the marke, using molecular sensitive approach today, because of the complexity of fecal density, vulnerability of stool to daily changes, and the presence of three sources of miRNAs in stool (cell-free from fecal homogenates, exsosomal miRNAs from fecal exosomes, and fecal colonocytes). To address these obstacles for developing a sensitive, economical and a non-invasive molecular colon cancer screening test, we have first carried out a microarray miRNA qualitative study, using Affymetrix GeneChip miRNA 2.0 Arrays, on immunocaptured and eniched stool colonocytes of 15 subjects [three healthy controls and twelve colon cancer patients [three TNM stage 0-1 (e.g., polyps $\geq 1$ $\mathrm{cm}$, villous or tubvillous, or with high grade dysplasia), three stage 2 , three stage 3, and three stage 4] in triplicates to select a smaller panel of 14 preferentially expressed mature miRNAs associated with colon cancer (12 Up-Regulated, miR-19a, miR-20a, miR-21, miR-31, miR34a, miR-96, miR-106a, miR-133a, miR-135b, miR-206, miR-224 and miR-302; and 2 Down-Regulated, miR-143 and miR-145). This was followed by an absolute quantitative digital PCR on these stool samples from the same stool samples. In which total small RNA extracted by immunocapture, followed by RT that employed TaqMan ${ }^{\star}$ miRNA Reverse Transcription (RT) Kit and a Custom TaqMan RT Primer

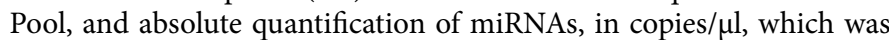
measured using a chip-based Absolute QuantStudio 3D Digital PCR analysis, to validate microarray results. To guarentee that we have used human and not bacterial small total RNA, we have carried out coextraction protocols with $E$. coli K1 strain RS18, compared Agilent electrophoretic patterns, and also sequenced random samples, using $\mathrm{mRNA} / \mathrm{miRNA}$ sequencing.

Our initial quantitative APCR miRNA data presented in this article, showed that the quantitative changes in the expression of a few mature miRNA genes in stool, which are associated with right and left colon cancer, would provide for a more convenient, sensitive and specific diagnostic screening markers than those tests currently available on the market, such as the low-sensitivity $(<15 \%)$ fecal occult blood test (FOBT); result in a better compliance; and is cheaper than the invasive and expensive colonoscopy exam, in a particular cancer, which can be cured if the cancer is detected at the early TNM stages, and that becomes uncurable and deadly if not diagnosed before it metastasizes.

Copyright: $(2018$ Ahmed FE. This is an open-access article distributed under the terms of the Creative Commons Attribution License, which permits unrestricted use, distribution, and reproduction in any medium, provided the original author and source are credited.
Test performance characteristics of the miRNA approach showed that the test has a high numerical predictive value in colon cancer. Moreover, underpinning of the miRNA markers as a function of total RNA showed that the test can numerically differentiate between control healthy subjects, and patients afflicted with colon cancer.

We propose to extend our initial results to a larger prospective and randomized five-years nested case-control study, to validate the expression of the above 14 miRNAs, in stool of 180 individuals in a study using [ 30 controls and 150 colon cancer patients [thirty precancerous polyps (stage $0-1$ ), forty five stage 2 , and seventy-five colon cancer stages 3 or 4] chosen randomly by an epidemiological method from 900 control and CC subjects to allow for proper randomization. Moreover, adequate number of patients would allow for statistically valid analysis, standardized test conditions, and provide a mean for determining the true sensitivity and specificity of a miRNA-screening approach in noninvasive human stool. Power-analysis has indicated that a total 180 individuals is an appropriate number of subjects to standardize and validate our proposed miRNA screening test. We may find out at the end of the proposed validation study in stool that fewer miRNAs, or even one miRNA, may suffice to serve as an efficient and a quantitative marker for the non-invasive diagnostic screening of colon cancer in human stool.

The above approach when combined with bioinformatics analysis, to correlate miRNA seed data with our previously published messenger (m)RNA target data in stool, allows for a thorough mechanistic understanding of how miRNA genes regulate mRNA expression, and would offer a better comprehensive diagnostic screening test for the non-invasive early detection stage (0-1) of colon cancer.

In order to show the clinical sensitivity and specificity of the proposed miRNA test, the absolute miRNA PCR values, in copies/ $\mu \mathrm{l}$, will be correlated with FOBT, colonoscopy, and pathology data. Standardization will establish test's performance characteristics (sample selection, optimal sample running conditions, preservation and storage) to ensure that the assay will perform the same way in any laboratory, by any trained personnel, anywhere. Ultimately, a smaller number of selected validated miRNAs $(<10)$ showing increased and reduced expression could suffice to give quantitative miRNAs colon cancer expression values. in Biotechnology, 2905 South Memorial Drive, Greenville, NC 278346222, USA, Tel. 252-689-5681, E-mail: gemtoxconsultants@yahoo.com

Received: April 07, 2018; Accepted: April 24, 2018; Published: April 27, 2018 